\title{
«Obtención, caracterización y diseño de una forma farmacéutica semisólida (ungüento) a base de quitosano con efecto cicatrizante»
}

\author{
«Obtención, caracterización y diseño de una forma \\ farmacéutica semisólida (ungüento) a base de \\ quitosano con efecto cicatrizante»
}

\author{
Lady C. Baltodano T., Juan E. Yaipen Ch. y César M. Fuertes R. (*)
}

\section{RESUMEN}

En el presente trabajo se estudió el efecto cicatrizante del quitosano, obtenido por desacetilación a partir de quitina de los caparazones del cangrejo de la variedad Cancer cetosus "Cangrejo Peludo", bajo la forma farmacéutica de ungüento a diferentes concentraciones. Para ello se aisló la quitina de los caparazones de cangrejos, se obtuvo el quitosano por desacetilación y se identificó por método espectrofotométrico (IR), solubilidad y determinando el grado de desacetilación mediante titulación potenciométrica y posteriormente se formuló el ungüento. Para la determinación del efecto cicatrizante se utilizó el test de cicatrización descrito por Howes y col, para heridas incisas, en el cual se emplearon 48 ratones albinos hembras de la especie Mus músculos de 1 mes y medio de edad, con un peso dentro del rango de 25 a $33 \mathrm{~g}$. Las muestras se administraron cada 12 horas por un periodo de 72 horas, al término del cual se cuantificó la resistencia que ofrecían las heridas tratadas, comparándolas con sus respectivos controles y se realizaron cortes histológicos para observar el grado de evolución histológica del proceso de cicatrización. El quitosano, obtenido por desacetilación a partir de quitina presenta mayor efecto cicatrizante bajo la forma farmacéutica de ungüento al 0,25\% (79,28\% efecto cicatrizante) en comparación con el grupo control (base del ungüento) se concluye que en las condiciones de ensayo el quitosano posee un significativo efecto cicatrizante.

\section{ABSTRACT}

In the present work the wound healing effect of the chitosan was studied, the chitosan obtained for desacetylation of chitin from the crab's shells of the variety Cancer cetosus «Shaggy Crab «, under the pharmaceutical form of unguent at different concentrations. For it there was isolated the chitin from the shell of crabs, the chitosan was obtained for desacetylation and identified by spectrophotometric method $(\mathrm{IR})$, solubility, determining the degree of desacetylatión by way of potentiometric qualifications and later the unguent was formulated. For the determination of the wound healing effect we used the test of wound healing described by Howes and col., for incision wounds; we used 48 female albino mice of the species Mus músculos of 1 month and a half of age, with a weight inside the range of 25 to $33 \mathrm{~g}$. The samples were administrated every 12 hours for a period of 72 hours., at the conclusion of the experiment the resistance that the treated wounds were offering, was quantified verifying them with their respective controls and histological cuts were realized to observe the degree of histological evolution of the process of wound healing. The chitosan, obtained by desacetylation from Chitin presents major wound healing activity under the pharmaceutical form of unguent at $0,25 \%(79,28 \%$ wound healing effect) in comparison with the group control (base of the unguent), it is concluded that under the test conditions chitosan possesses a significant healing wound effect.

(*) Instituto de Investigación en Ciencias Farmacéuticas y Recursos Naturales «Juan de Dios Guevara» Facultad de Farmacia y Bioquímica-Universidad Nacional Mayor de San Marcos-PERÚ 


\section{INTRODUCCIÓN}

La producción mundial de crustáceos (cangrejo, camarón, langosta, etc.) se ha incrementado enormemente en los últimos años. En general solamente el 20 a $30 \%$, del peso vivo de estas especies de crustáceos es utilizado para la alimentación humana; el resto constituido por vísceras y exoesqueleto, considerados como contaminantes ambientales (desechos), constituyen compuestos de valor comercial no aprovechados tales como quitina, proteínas, pigmentos y minerales.

Debido a la variabilidad de fuentes de materias primas de caparazones de crustáceos y métodos de obtención de quitina es oportuno realizar este trabajo con el fin de obtener productos de interés comercial, y tecnología transferible a los sectores productivos del país.

Los derivados de quitina tienen un inmenso campo de aplicación con relevante valor económico, así el uso en la industria alimentaría involucra importantes volúmenes, en la industria farmacéutica para la preparación de productos de liberación prolongada, entre otros. La búsqueda de nuevos biomateriales con propiedades específicas, es un campo de interés científico y tecnológico que orienta los esfuerzos de muchos centros de investigación. La utilización en la medicina, industria textil, la agricultura y el tratamiento de aguas como bioremediador, son ejemplos de sus vastas aplicaciones.

El presente trabajo trata de satisfacer de algún modo las necesidades de salud de nuestro país mediante la transformación de caparazones de cangrejos, tan abundantes en nuestro país, en un producto farmacéutico eficaz en el proceso reparador de la piel, siendo el objetivo general: Obtener, caracterizar el quitosano a partir de la quitina y diseñar una forma farmacéutica semisólida (ungüento), de aplicación tópica con efecto cicatrizante y como objetivos específicos: Extraer y aislar quitina, Obtener quitosano a partir de la quitina, Caracterizar la quitina y quitosano, Determinar el grado de desacetilación del quitosano, Comprobar el efecto cicatrizante de quitosano bajo una forma farmacéutica.

\section{EXPERIMENTAL}

\section{DEL ESTUDIO QUÍMICO:}

\section{Obtención de la quitina}

Los Cangrejos de la Variedad «peludo» Cancer cetosus se adquirieron en el Terminal pesquero de Ventanilla ubicado en el Callao. Los cangrejos fueron separados de sus caparazones, para luego ser lavados de manera acuciosa con abundante agua, separando los restos orgánicos que pudieran estar presentes. Posteriormente se desinfectaron con una solución de hipoclorito de sodio, lavados con agua y finalmente ser llevado a una estufa por 48 horas hasta peso constante. Los caparazones secos se sometieron a reducción de tamaño de partícula.

El polvo obtenido se sometió a desmineralización de ácido clorhídrico $2 \mathrm{~N}$, se sometio a desproteinización con una solución de hidróxido de sodio $2 \mathrm{~N}$ primero a temperatura ambiente y luego a baño Maria bajo agitación constante para luego ser lavado con alcohol y finalmente secado en una estufa por 12 horas.Luego sigue la etapa de decoloracion y luego de lavado hasta neutralidad. La muestra finalmente se desecó en estufa con flujo de aire a $60 \stackrel{\circ}{C}$ por 12 horas.

\section{Obtención del quitosano}

La quitina obtenida, se sometió al proceso de desacetilación, con una solución de hidróxido de sodio y a una temperatura de $120{ }^{\circ} \mathrm{C}$ con agitación constante luego se lava con abundante agua hasta neutralidad y finalmente secado en estufa a una temperatura de $60 \stackrel{\circ}{\circ}$ por 24 horas.

\section{Caracterización del quitosano}

\section{Método espectrofotométrico. (IR)}

La quitina y quitosano fueron identificados mediante espectroscopia infrarroja (IR), utilizando un espectrofotómetro PERKIN ELMER FT-IR Modelo: Spectrum 1000. Los espectros obtenidos se interpretaron y compararon. 


\section{Solubilidad}

Ensayos de Solubilidad: en 5 tubos de ensayo se colocó una pequeña porción de la muestra (100mg) y se le agrego $5 \mathrm{~mL}$ del solvente respectivo: Agua, etanol, ácido acético, ácido oxálico y ácido clorhídrico, se agitó, se dejó en reposo por 24 horas y se observan los resultados.

\section{Determinación del grado de desacetilación (dga):}

Método: Valoración titulométrica de los grupos aminos. [1]

Para la determinación del contenido de grupos amino de la muestra de quitosano en una solución de ácido clorhídrico luego se tituló con una solución de hidróxido de sodio .La valoración se llevó a cabo midiendo el cambio de pH cada $2 \mathrm{~mL}$ de base añadida, la adición se realizó de forma lenta y con agitación continua para homogenizar la solución y evitar errores debido a la posible precipitación del polímero. Las mediciones se realizaron 3 veces por muestra.La diferencia entre los dos puntos de inflexión en la curva de titulación corresponde a la cantidad de ácido requerido para protonar los grupos aminos del quitosano, la concentración de éstos se determina utilizando la expresión:

$$
\% N H 2=\frac{16.1(\mathrm{Y}-\mathrm{X}) \times \mathrm{F}}{(\text { Fórmula } 1)}
$$

W

Donde:

«Y»= Punto de inflexión mayor.

«X» = Punto de inflexión menor.

Ambos expresados como volúmenes. «F» = Molaridad de la solución de $\mathrm{NaOH}$. «W» = Peso en gramos de la muestra 16.1 = Valor relacionado con el peso equivalente del quitosano.

\section{Diseño de la forma farmacéutica (ungüento) de aplicación tópica a base de quitosano}

Desarrollar una forma farmacéutica tópica (ungüento) estable a base de quitosano que conserve el efecto cicatrizante.
Para el diseño se tomo como principal referencia que la forma farmacéutica a elaborar seria utilizada tópicamente como cicatrizante, por tal motivo esta debería contar con poder de penetración a través de la piel, por lo que se pensó en una forma farmacéutica semisólida como un gel o ungüento.Dadas las propiedades físico-químico del quitosano obtenido y la solubilidad se optó por diseñar un ungüento con una base hidrocarbonada la cual está representada por la vaselina blanca y el ungüento blanco, la que permite incorporar una cantidad mínima de componente acuoso. Sirven para mantener los medicamentos en contacto prolongado con la piel y actúan como vendaje oclusivo.Las bases hidrocarbonadas se usan, principalmente, por sus efectos emolientes y son difíciles de eliminar. No se «secan» ni se modifican en forma notoria con el envejecimiento. Son muy recomendables en el proceso de cicatrización de heridas.

\section{DEL ESTUDIO FARMACOLÓGICO:}

Determinación del efecto cicatrizante de los ungüentos a base de quitosano por el método de la fuerza de tensión (Método de Howes y col.) [2]

Determinar el efecto cicatrizante del ungüento a base de quitosano en comparación con un control (Cicatrin $\left.{ }^{\circledR}\right)$ por el método de la fuerza de tensión.

Es un estudio experimental pre-clínico se realizó en ratones.

Tabla № 1 Distribución de los grupos experimentales

\begin{tabular}{|l|c|c|c|c|c|c|}
\hline & \multicolumn{5}{|c|}{ Grupos experimentales } & \multirow{3}{*}{ Blanco } \\
\hline \multirow{3}{*}{ MÉTODO } & \multicolumn{3}{|c|}{$\begin{array}{c}\text { Quitosano en } \\
\text { ungüento }\end{array}$} & \multicolumn{2}{|c|}{ Control } & \\
\cline { 2 - 7 } & $\begin{array}{c}1 \\
\%\end{array}$ & $\begin{array}{c}0.50 \\
\%\end{array}$ & $\begin{array}{c}0.25 \\
\%\end{array}$ & $\begin{array}{c}\text { Base del } \\
\text { Ungüento }\end{array}$ & Cicatrin & \\
\hline TENSIOMETRICO & 6 & 6 & 6 & 6 & 6 & 6 \\
\hline HISTOLOGICO & 2 & 2 & 2 & 2 & 2 & 2 \\
\hline TOTAL & 8 & 8 & 8 & 8 & 8 & 8 \\
\hline
\end{tabular}

Alimentación: Dichos animales se mantuvieron durante todo el estudio experimental con alimentación y agua «ad libitum»

\section{Metodología}

Fundamento: El método de la Fuerza de Tensión mide la fuerza necesaria para abrir una 
herida incisa de $1 \mathrm{~cm}$ de largo, realizada en el tercio anterior del lomo y perpendicular al eje longitudinal de un ratón.

\section{- Ambientación o acondicionamiento}

Los 48 ratones albinos hembras, cepa Balb/c/ CNPB provenientes del Bioterio del Centro Nacional de Producción de Biológicos (INS), fueron distribuidos al azar en 6 grupos de 8 ratones cada grupo y colocados en jaulas individuales. Se mantuvieron en observación por un periodo de una semana (7 días), verificándose la condición óptima de los ratones para el estudio. A dichos animales se les mantuvo en un ambiente ventilado apropiadamente, en jaulas individuales, con alimento balanceado (INS) y agua Ad-libitum, por un periodo de 7 días previos al inicio de la experiencia. Se procuró un ciclo de luz/ oscuridad de 12 cada uno por día.

\section{- Depilación}

Después de una semana de ambientarse los ratones al lugar de trabajo, se procedió a depilarlos con crema depilatoria Veet $\AA$ en el primer tercio dorsal anterior en un área aproximada de $2 \mathrm{~cm} 2$, se anestesió con pentobarbital sódico por vía subcutánea a una concentración de $50 \mathrm{mg} / \mathrm{Kg}$ de peso. La depilación se realizó previa humectación (con agua tibia) de la zona a depilar, luego se agrega la crema depilatoria Veet@ la cual permaneció 3 minutos para ejercer su efecto. Finalmente con la ayuda de gasas húmedas se retiró la crema. Posterior a la depilación, los ratones se colocaron en sus respectivas jaulas individuales teniendo estos libre acceso a bebida y comida. La depilación se realizó 24 horas antes del procedimiento quirúrgico a fin de descartar cualquier reacción alérgica a la crema depilatoria.

\section{- Incisiones y sutura}

Después de 24 horas de la depilación, se procedió a anestesiar a los ratones administrándoles una dosis de $50 \mathrm{mg} / \mathrm{Kg}$ de pentobarbital sódico vía subcutánea (tercio inferior del lomo. Se colocó al ratón sobre la mesa de trabajo, desinfectando el área depilada y marcando 2 puntos equidistantes en $1 \mathrm{~cm}$ y perpendicular al eje longitudinal del ratón (zona de corte). Se realizó el corte sobre la zona indicada (cicatriza de primera intención), se unieron los bordes con un punto de sutura con hilo en la parte central del corte. Esta etapa se realizó cumpliendo condiciones de asepsia.

\section{- Aplicación}

Obtenido el punto de sutura, se administró en forma tópica la primera dosis del tratamiento sobre la incisión con la ayuda de un hisopo, logrando obtener una distribución homogénea sobre la incisión. Se aplicaron tanto las formulaciones a base de quitosano, a los controles: la base del ungüento y el Cicatrin $\AA$ en sus respectivos grupos, mientras que el blanco no recibió tratamiento. Se repitió el tratamiento cada 12 horas en un lapso de 72 horas.

\section{- Determinación de la fuerza de tensión}

Pasadas las 72 horas de tratamiento, se procedió a realizar la medición de la fuerza de tensión en el equipo de tensión. Para ello los ratones fueron previamente anestesiados con una dosis de $100 \mathrm{mg} / \mathrm{Kg}$ de pentobarbital sódico vía intraperitoneal. Luego se colocaron en la mesa de trabajo y se procedió a marcar los puntos donde se engancharan las agujas del equipo de tensión, a $0,5 \mathrm{~cm}$ de ambos extremos del punto de sutura. Se retiró con mucho cuidado el punto de sutura y colocó al animal en posición en decúbito ventral sobre el aparato de tensión, luego se insertó las agujas, retirando las base del recipiente y de inmediato se abrió la bureta para dejar caer el agua hasta que se generó una fuerza de tensión capaz de abrir la herida en toda su longitud. Finalmente, se anotó el nivel de agua requerido

Usando los datos obtenidos por el Método de Fuerza de Tensión según Howes y col., se determinó el porcentaje de efecto cicatrizante utilizando la siguiente fórmula:

$\%$ Efecto cicatrizante de la muestra $=$

$$
\frac{(\mathrm{mL} \text { Muestra }-\mathrm{mL} \text { Control }) \times 100}{\mathrm{~mL} \text { Control }}
$$

\section{Determinación del efecto cicatrizante de los ungüentos a base de quitosano por el método del corte histológico.}

Corroborar los resultados obtenidos en el método tensiométrico y observar la evolución macroscópica y microscópica de la cicatriz. 


\section{Corte Histológicos.}

Pasadas las 72 horas de tratamiento, se procedió a realizar los cortes histológicos. Los ratones fueron previamente sacrificados con una dosis de $100 \mathrm{mg} / \mathrm{Kg}$ de pentobarbital sódico vía intraperitoneal, las muestras de tejidos con cicatrices experimentales fueron obtenidos inmediatamente a la muerte de los animales, seleccionando un área de $2 \mathrm{~cm}$ de ancho por $2.5 \mathrm{~cm}$ de largo, estos tejidos fueron sujetados cuidadosamente en trozos de tecnopor, para evitar el enrollamiento natural del tejido separado, y depositados en una solución de formaldehído amortiguado neutro al $10 \%$ para lograr la fijación que conserva una imagen del tejido, como si estuviera vivo, luego viene la deshidratación con soluciones de concentraciones crecientes de etanol y el aclaración del tejido (se vuelve transparente) con xilol, después, para poder hacer los cortes se realiza la infiltración o inclusión en parafina, formándose un bloque solidó, la etapa siguiente es el corte que se realiza en un micrótomo, a continuación se hace el montaje en una lamina portaobjetos cubierta con material adherente (albúmina de huevo), luego se retira la parafina restante con xileno, después se realizó la tinción en este caso con hematoxilina-eosina, después se rehidrata con soluciones de concentraciones decrecientes de etanol, de modo que pueda fijarse con un medio de montaje como el bálsamo de Canadá, que tiene índice de refracción similar al vidrio, se cubre con un cubreobjetos y se sella con esmalte transparente.[3] (Dicho ensayo lo realizó el Departamento de Histopatológica del Hospital Nacional Arzobispo Loayza).

Teniendo los cortes histológicos fijos en placas portaobjetos, se realizó las observaciones en el microscopio.

\section{RESULTADOS}

\section{DEL ESTUDIO QUÍMICO:}

Tabla № 2. Rendimiento de quitosano obtenido a partir de quitina por método químico

\begin{tabular}{|l|l|}
\hline Quitosano & (\%) \\
\hline En relación a la muestra total & $7.02 \%$ \\
\hline En relación a la quitina & $61.78 \%$ \\
\hline
\end{tabular}

\section{Caracterización del Quitosano.}

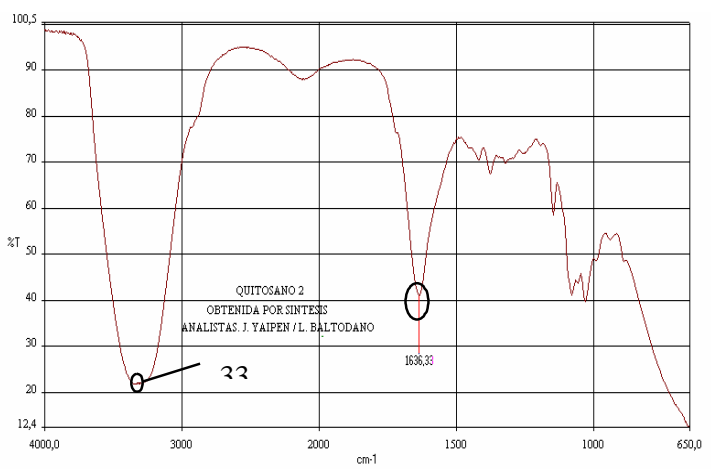

Figura № 1 Espectro infrarrojo del quitosano en el rango de $4000-650 \mathrm{~cm}^{-1}$

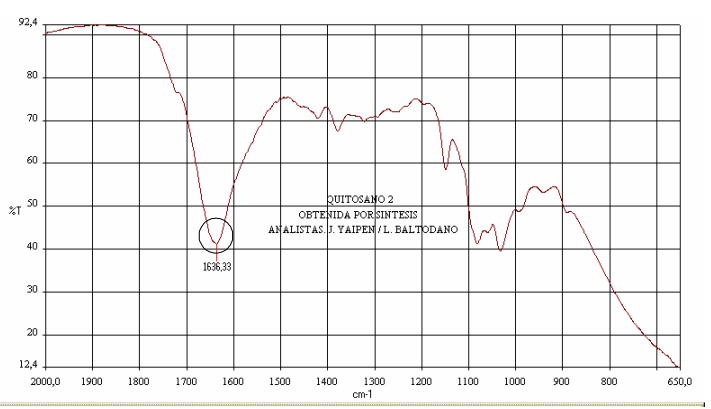

Figura № 2 Espectro infrarrojo del quitosano en el rango de 2000-650 $\mathrm{cm}^{-1}$

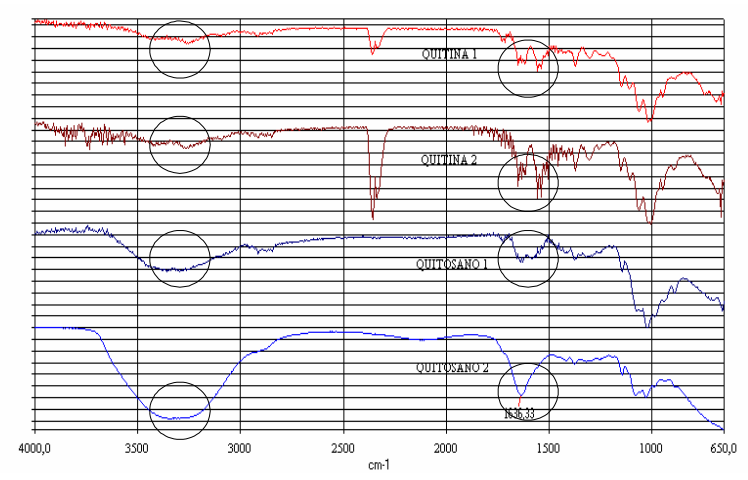

Figura № 3 Comparación de los espectros infrarrojo de la quitina y quitosano en el rango $4000-650 \mathrm{~cm}^{-1}$

Solubilidad.

Tabla № 3 Determinación de la solubilidad de la quitina y el quitosano

\begin{tabular}{l|l|l|l|l|l|}
\hline & AGUA & ALCOHOL & $\begin{array}{l}\text { ÁCIDO } \\
\text { ACÉTICO }\end{array}$ & $\begin{array}{l}\text { ÁCIDO } \\
\text { OXÁLICO }\end{array}$ & $\begin{array}{l}\text { ÁCIDO } \\
\text { CLORHI- } \\
\text { DRICO }\end{array}$ \\
\hline QUITINA & - & - & - & - & - \\
\hline QUITOSANO & - & - & +++ & ++ & ++ \\
\hline$-\quad$ INSOLUBLE \\
$+\quad$ MIGERAMENTE SOLUBLE \\
++ \\
++
\end{tabular}


Determinación del grado de desacetilación por titulación potenciométrica

Peso: $0.1041 \mathrm{~g}$

Solución valorante $\mathrm{NaOH} 0.1 \mathrm{~N}(\mathrm{~F}=0,99725)$

CÁLCULOS:

$\% \mathrm{NH2}=16.1(\mathrm{Y}-\mathrm{X}) \times \mathrm{F} / \mathrm{W} \quad$ (Muestra 1)

$\% \mathrm{NH} 2=16.1(6) \times 0.099725 / 0.1041 \mathrm{~g}$

$\% \mathrm{NH} 2=92.54 \%$

Peso: $0.1076 \mathrm{~g}$

Solución valorante $\mathrm{NaOH} 0.1 \mathrm{~N}(\mathrm{~F}=0,99725)$

CÁLCULOS:

$\% \mathrm{NH} 2=16.1(\mathrm{Y}-\mathrm{X}) \times \mathrm{F} / \mathrm{W} \quad$ (Muestra 2)

$\% \mathrm{NH} 2=16.1(6,5) \times 0.099725 / 0.1076 \mathrm{~g}$

$\% \mathrm{NH} 2=97.00 \%$

Peso: $0.1064 \mathrm{~g}$

Solución valorante $\mathrm{NaOH} 0.1 \mathrm{~N}(\mathrm{~F}=\mathbf{0 , 9 9 7 2 5 )}$

CÁLCULOS:

$\% \mathrm{NH} 2=16.1(\mathrm{Y}-\mathrm{X}) \times \mathrm{F} / \mathrm{W} \quad$ (Muestra 3)

$\% \mathrm{NH} 2=16.1(6,5) \times 0.099725 / 0.1064 \mathrm{~g}$

$\% \mathrm{NH} 2=98.00 \%$

Tabla № 4 Resultados de la determinación del grado de desacetilación por titulación potenciométrica del quitosano

Del estudio farmacológico.

\begin{tabular}{|l|l|l|l|}
\cline { 2 - 4 } \multicolumn{1}{c|}{} & Muestra 1 & Muestra 2 & Muestra 3 \\
\hline$\%$ NH2 & 92.54 & 97.00 & 98.00 \\
\hline $\begin{array}{l}\% \mathrm{NH} 2 \\
\text { promedio }\end{array}$ & 95.85 & \multicolumn{2}{|l}{} \\
\hline
\end{tabular}

Determinación del efecto cicatrizante de los ungüentos a base de quitosano por el método de la fuerza de tensión (Método de Howes y col.)

Tabla № 5 Resultados de la determinación del efecto cicatrizante de los ungüentos a base de quitosano frente al grupo control (Base del ungüento)

\begin{tabular}{|l|l|l|c|}
\cline { 2 - 4 } \multicolumn{1}{c|}{} & \multicolumn{1}{c|}{ Tratamiento } & $\begin{array}{c}\text { Volumen } \\
(\mathbf{m L})\end{array}$ & $\begin{array}{c}\text { \% Efecto } \\
\text { cicatrizante }\end{array}$ \\
\hline \multirow{3}{*}{ Muestras } & $0,25 \%$ Quitosano & 74,08 & 79,28 \\
\cline { 2 - 4 } & $0,5 \%$ Quitosano & 47,65 & 15,32 \\
\cline { 2 - 4 } & $1 \%$ Quitosano & 53,90 & 30,45 \\
\hline \multirow{2}{*}{ Control } & Cicatrin & 40,47 & $-2,06$ \\
\cline { 2 - 4 } & Base del ungüento & 41,32 & 0,00 \\
\hline Blanco & Sin tratamiento & 33,38 & \multicolumn{2}{|c}{} \\
\hline
\end{tabular}

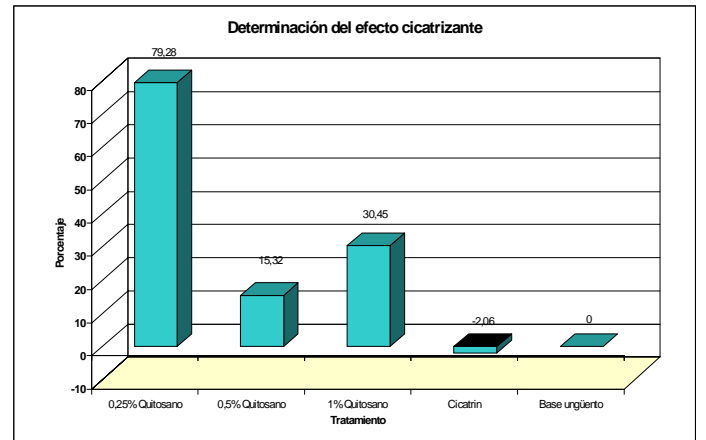

Figura № 4 Determinación del efecto cicatrizante

Determinación del efecto cicatrizante de los ungüentos a base de quitosano por el método del corte histológico.

Corte histológico de cicatriz experimental tratada con ungüento a base de quitosano al $1.0 \%$

Se observa que el tejido epidérmico ha formado una solución de continuidad con escasa fibrina (costra) el lecho de la lesión se encuentra en vías de completar su reepitelización, se evidencia la presencia de fibroblastos (como células alargadas con núcleos pequeños y oscuros) y de tejido colágeno, caracterizados por la coloración sonrosadas.

Corte histológico de cicatriz experimental tratada con ungüento a base de quitosano al $0.5 \%$

Se observa que la cicatrización presenta tejido de granulación, la epidermis se encuentra en solución de continuidad con reepitelización incompleta. El lecho de la lesión es discontinuo con infiltración mononuclear y macrófagos en número de 40 por campo. Se evidencia la presencia de fibroblastos en la dermis profunda

Corte histológico de cicatriz experimental tratada con ungüento a base de quitosano al $0.25 \%$

Se observa que el tejido epidérmico ha formado una solución de continuidad con fibrina (costra) en la superficie, en la epidermis se observa reepitelización, asimismo se evidencia la presencia de tejido fibroso ( fibroblastos) y tejido conjuntivo ( colágeno) en el lecho de la lesión. Hay infiltración mononuclear en número de 20 macrófagos por campo. 


\section{Corte histológico de cicatriz experimental tratada con base del ungüento (Vaselina)}

Se observa reepitelización, tejido de granulación subepitelial y fibroblastos.

\section{Corte histológico de cicatriz experimental

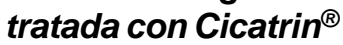

Se observa una epidermis delgada con solución de continuidad con pared costrosa y tejido fibroso (fibroblastos), se observa reepitelización, en el lecho de la lesión se observa con solución de continuidad con gran infiltración mononuclear, cicatrización incipiente

\section{Corte histológico de cicatriz experimental sin tratamiento}

Se observa una epidermis delgada con solución de continuidad con tejido de granulación insuficiente, no hay reepitelización, existe incipiente proliferación y migración celular

\section{DISCUSIÓN DE RESULTADOS}

Como paso previo a la caracterización y diseño de la forma farmacéutica de aplicación tópica, se efectúo el aislamiento de la quitina a partir de los caparazones de cangrejos de la variedad Cancer cetosus «Cangrejo Peludo" y obtención de quitosano a partir de ella.

El método químico empleado permite la remoción de una manera efectiva de las sustancias que acompañan a la quitina en la materia prima el grado de pureza se evidencia mediante la espectroscopia infrarroja.

La obtención del quitosano por método químico a partir de la quitina alcanzó un rendimiento de $7,02 \%$ de quitosano con respecto a la muestra total y de $61,78 \%$ con respecto a la quitina (Tabla № 2). El método químico empleado para la obtención de quitosano permitió obtener un producto con unas características fisicoquímicas aceptables (identificación por IR, solubilidad, grado de desacetilación) en comparación con otros estudios realizados con diferentes especies $[4,5]$ la cual se puede evidenciar en el espectro infrarrojo de quitosano.(Figura №1,2).

La caracterización de quitina y quitosano por el método de espectroscopía Infrarrojo se basa principalmente en la comparación de los espectros relacionados con la presencia 0 ausencia del grupo carbonilo y el radical acetilo. La quitina presenta un espectro con una banda a $1650 \mathrm{~cm}^{-1}$ que corresponde a la vibración del grupo carbonilo y una vibración secundaria del grupo amino secundario con una banda de $1603,95 \mathrm{~cm}^{-1}$; el espectro de quitosano presenta una banda a $1636,33 \mathrm{~cm}^{-1}$ que se debe a la presencia de grupo amino primario $[6,7]$.

En el espectro de quitosano se observa una banda muy pronunciada a $3315,79 \mathrm{~cm}^{-1}$ probablemente del resultado de acumulación del grupo oxidrilo y amino primario.

La solubilidad de la quitina y quitosano nos permite confirmar las características propias de estos compuestos y así poderlos identificarlos. En los ensayos de solubilidad se observó que la quitina es insoluble en agua, ácidos orgánicos (Ácido acético, ácido oxálico) e inorgánicos diluidos (Ácido clorhídrico) esto debido a los grupos acetilos que presenta la quitina, mientras que el quitosano es insoluble en agua pero presenta solubilidad en ácidos orgánicos (Ácido acético, ácido oxálico) e inorgánicos diluidos (Ácido clorhídrico) debido a la protonación de los grupos aminos $\left(-\mathrm{NH}_{2}\right)$ en amonio $\left(-\mathrm{NH}_{3}{ }^{+}\right)[3]$ (Tabla № 3).

El grado de desacetilación con el fin de conocer el contenido de grupos aminos en las muestras de quitosano se determinó por titulación potenciométrica donde se produce una curva de titulación con dos puntos de inflexión cuyos valores se determinaron según el criterio de la primera derivada. La diferencia entre los dos puntos de inflexión en la curva de titulación corresponden a la cantidad de ácido requerido para protonar los grupos aminos del quitosano el análisis se realizo por triplicado donde se observa que la proporción de los grupos aminos libres es alta $(95.85 \%)$ lo que es indicativo que el método utilizado permitió la obtención de un producto altamente desacetilado (Tabla № 4). Los resultados son congruentes con los obtenidos previamente por espectroscopia infrarroja que indica la desacetilación de la quitina.

Para la preparación de la forma farmacéutica tópica a base de quitosano, se selecciono una base hidrocarbonada: la vaselina sólida, debido a que permite un contacto prolongado con la piel actuando como un vendaje oclusivo; 
Asimismo, por ser una sustancia químicamente inerte en comparación con las bases de geles como Carbomer 940, Carboximetilcelulosa y Sepigel las cuales presentan incompatibilidad con el quitosano debido a los grupos aminos libres.

Las concentraciones elaboradas a base de quitosano fueron de $0.25 \%, 0.50 \%$ y $1.0 \%$ los cuales fueron establecidas de acuerdo a la propiedades fisicoquímicas del quitosano especialmente a su solubilidad en un medio acuoso y a la capacidad de la vaselina de permitir incorporar agua en su composición obteniéndose una forma farmacéutica estable en el tiempo. $[8,9,10]$

En el estudio del efecto cicatrizante de los ungüentos de quitosano por el método de la fuerza de tensión, se determinó que el porcentaje de efecto cicatrizante máximo fue de $79.28 \%$ a la concentración de $0.25 \%$ de Quitosano seguido por los ungüentos a la concentración de $1 \%$ y $0.50 \%$ de quitosano $(30.45 \%$ y $15.32 \%$ respectivamente) en comparación con el grupo control (base del ungüento) (Tabla № 5).

En el análisis estadístico de los valores medios de fuerza necesaria para abrir lesiones cicatrizadas en ratones según porcentajes de quitosano ( $\mathrm{mL}$ de agua empleados), el error estándar o coeficiente de variación máximo es de $3 \%$ indicando que los datos obtenidos de las medias son confiables.

En el análisis de varianza de los datos de fuerza necesaria para abrir lesiones cicatrizadas de ratones tratadas con los ungüentos, compara las medias de los 5 grupos experimentales definidos por la dosis, obteniéndose un $\mathrm{p}<$ 0.001 ; con lo cual podemos afirmar que hay diferencia significativa entre las medias de los grupos de quitosano a diferentes concentraciones.

Para el análisis de múltiples comparaciones de la media mediante la prueba de LSD, se hizo uso del programa SPSS, versión 13, 2004, el cual nos permitió identificar los tratamientos estadísticamente significativos y eliminando aquellos que no los son.

Asimismo podemos observar que no hay diferencia significativa entre la base del ungüento (vaselina) y el cicatrin. El cicatrin es una combinación de antibióticos que favorecen indirectamente en el proceso de cicatrización mediante su acción antibacteriana, mientras que la base del ungüento (Vaselina) por sus propiedades oclusivas evita la exposición de la herida con el medio ambiente En ambos casos se observa que el «efecto cicatrizante» que presentan es debido a estas propiedades, no atribuyéndoles actividad cicatrizante propiamente dicha.

La cicatrización también fue evaluada por un estudio histológico, debido a la relación existente entre el aumento de la fuerza de tensión y la evolución positiva histológica del tejido. En la observación microscópica de los cortes histológicos, se demostró que el ungüento al $0.25 \%$ presenta una evolución de la cicatrización mas avanzada la cual se manifiesta principalmente en el aumento de células basales epidérmicas, observándose la formación de una solución de continuidad con fibrina (costra) en la superficie, reepitelización, presencia de tejido fibroso (fibroblastos) y tejido conjuntivo (colágeno). También se observó que la cantidad de linfocitos es menor debido al inicio de la fase de fibroplasia, que indica el final de la fase inflamatoria, observándose la formación de tejido conectivo laxo, confirmando la presencia de fibroblastos y angiogénesis.

\section{CONCLUSIONES}

- La caracterización de la quitina, aislada de los caparazones del "Cangrejo peludo" Cancer cetosus, y el quitosano, obtenido por desacetilación química de la quitina, cumplen con las propiedades fisicoquímicas, de solubilidad, grado de desacetilación y espectroscopia IR.

- El método de obtención de quitosano permitió obtener un producto altamente desacetilado ( $95.85 \%$ )

- Se comprobó que el quitosano bajo la forma farmacéutica semisólida (ungüento) presenta actividad terapéutica como cicatrizante externo. El ungüento con mayor eficacia en el tratamiento fue el de $0.25 \%$ a base de quitosano con un $79.28 \%$, seguido por el ungüento al $1 \%$ con $30.45 \%$, el ungüento al $0.50 \%$ con $15.32 \%$, tomando como valor referencial al grupo control.

- Los estudios histológicos demuestran que los ungüentos a base de quitosano estimulan 
la proliferación y migración celular siendo el ungüento al $0.25 \%$ el que presenta mejores resultados.

\section{RECOMENDACIONES}

- Promover la transformación de desechos marinos en productos con actividad biológica.

- Continuar los estudios clínicos de la actividad terapéutica como cicatrizante externo del ungüento a base de quitosano.

- Continuar con el estudio y evaluación de la actividad cicatrizante de quitosano obtenido de otras especies marinas peruanas.

\section{AGRADECIMIENTOS}

Expresamos nuestros más sinceros agradecimientos:

A Dios, quien nos ilumina y guía en todo momento.
A nuestra Alma Mater y en especial a la Facultad de Farmacia y Bioquímica por nuestra formación profesional y permitirnos ser parte de ella.

Nuestro Profesor Guía Dr. Cesar M. Fuertes Ruitón, por su amistad, paciencia y su constante apoyo durante el desarrollo de esta tesis.

A nuestros profesores de la Facultad de Farmacia y Bioquímica - UNMSM, por sus aportes académicos y amistad.

A las cátedras de: Química Orgánica, Farmacognosia, Farmacotecnia y Farmacología, por su cooperación para la realización de esta tesis.

Al Departamento de Histopatólogia del Hospital Arzobispo Loayza.

Asimismo agradecemos a aquellas personas amigas, familiares que mediante su apoyo brindado y motivación hicieron posible este logro.

De igual forma deseamos expresar nuestro agradecimiento al Jurado Examinador y Calificador de esta tesis por sus sugerencias.

\section{REFERENCIAS BIBLIOGRÁFICAS}

[1] Parada et al. Caracterización de quitosano por viscosimetría capilar y valoración potenciométrica. Revista Iberoamericana de Polímeros.2004; 5[1]1-3.

[2] Howes E, Sooy J, Harvey S. The healing of wound as determined by their tensile strength J.A.M.A. [The Journal of the American Medical Association] 1929; 42[5].

[3] Guillermo R. Comprobación del Efecto Cicatrizante de Peperomia scutellaefolia R. et P., Aspectos Etnofarmacológicos, Botánicos y estudio Químico. Tesis para optar al Título de Químico Farmacéutico. 2002 UNMSM. Lima

[4] Mármol Z, Gutiérrez E, Páez G, Ferrer J, Rincón M. [base de datos en internet] Desacetilación termoalcalina de quitina de conchas de camarón.[citado 10 ene 2006] multiciencias4[2]completa. Disponible en: http:// www.serbi.luz.edu.ve/pdf/mc/v4n2/art_03.pdf

[5] Raymond L, Morin F, Marchessault R. Degree of deacetylation of chitosan using conductometric titration and solid-state NMR. Carbohydrate Research. 1993;246:331-336

[6] Socrates, G. Infrared Characteristic Group Frequencies: Tables and Charts, $3^{\mathrm{a}}$ ed., Wiley, 2001.

[7] Mármol Z, Gutiérrez E, Páez G, Ferrer J, Rincón M. [base de datos en internet] Desacetilación termoalcalina de quitina de conchas de camarón. [citado 10 ene 2006] multiciencias4[2]completa. Disponible en: http:// www.serbi.luz.edu.ve/pdf/mc/v4n2/art_03.pdf

[8] Gouda I, Larm O, Inventores, Medicarb AB, Assignee, Method Of Promoting Dermal Wound Healing With Chitosan And Heparin Or Heparin Sulfate. United States patent US5902798 1999 Mayo 11

[9] Trillo, F. tratado de Farmacia Galenita. Editorial Egraf S.A. 1er Edicion. Madrid 1996.

[10] Convención de la Farmacopea de los Estados Unidos de América. Farmacopea de los Estados Unidos de América: formulario nacional. 2006. USP 29 NF 24.

E-mail:

Q.F Lady Baltodano ladyfyb@yahoo.es

Q.F. Juan Yaipen juyachi@yahoo.es

Mg. Cesár Fuertes cfuertesr@unmsm.edu.pe 